\title{
Fetal growth trajectory in type 1 pregestational diabetes (PGDM) — an ultrasound study
}

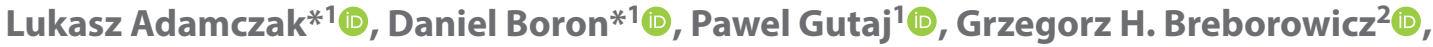 \\ Jerzy Moczko3 ${ }^{3}$, Ewa Wender-Ozegowska ${ }^{1}(\mathbb{D}$ \\ ${ }^{1}$ Department of Reproduction, Chair of Obstertics, Gynecology and Gynecological Oncology, \\ Poznan University of Medical Sciences, Poland \\ ${ }^{2}$ Department of Gynecological Oncology, Chair of Obstertics, Gynecology and Gynecological Oncology, Poznan, Poland \\ ${ }^{3}$ Department of Computer Science and Statistics, Poznan University of Medical Sciences, Poland
}

*equal contribution

\begin{abstract}
Objectives: Growth disorders are frequent in diabetic pregnancies. However, they are difficult to predict and capture early during pregnancy. These newborns are at risk of obesity, diabetes, and cardiovascular disease. While developing, fetal growth abnormalities are typically progressive. Therefore, capturing the earliest moment when they emerge is essential to guide subsequent obstetric management.

Material and methods: We aimed to analyze fetal ultrasound growth trajectories in type 1 diabetics. Moreover, we aimed to establish time points when first ultrasound manifestations of fetal growth abnormalities appear and to identify factors that affect fetal growth in women with diabetes.

We collected clinical and ultrasound data from 200 patients with PGDM managed in the third-referential centre for diabetes in pregnancy. During every visit, patients underwent an ultrasound examination according to a standard protocol giving 1072 ultrasound scan's records. Every ultrasound consisted of fetal weight estimation, according to the Hadlock 3 formula. Retrospectively patients were divided into three groups depending on neonatal weight. In the group of 200 patients, 60 (30\%) delivered LGA and 9 (4.5\%) SGA newborns.

Results: Fetal growth trajectories show different patterns among fetuses with growth abnormalities in women with type 1 diabetes. The moment, when fetal growth curves diverge, seems to take place in the second trimester, just after the $23^{\text {rd }}$ week of gestation.

Conclusions: It suggests that fetal growth abnormalities in type 1 diabetes may have its roots much earlier than expected. In the first trimester, there were differences in LDL-cholesterol, total cholesterol, triglyceride levels and in insulin requirements between AGA, SGA and LGA subgroups.
\end{abstract}

Key words: fetal growth; pregestational diabetes; ultrasound; type 1 diabetes mellitus

Ginekologia Polska 2021; 92, 2: 110-117

\section{INTRODUCTION}

Pregnancy in diabetic patients increases the risk of developing maternal and fetal complications. Pregestational diabetes mellitus (PGDM) complicates about 0.2-0.3\% of pregnancies, depending on the studied population [1]. Patients with diabetes are much more likely to develop obstetric complications such as pregnancy-induced hypertension, preeclampsia and polyhydramnios. Premature births, operational deliveries, perinatal infections are also much more common in that group of patients [2-4]. Acute carbohydrate disturbances may pose a direct threat to the life of the pregnant woman and fetus.
Among fetal complications we can distinguish congenital malformations, neonatal hypoglycemia and in particular excessive growth of the fetus (LGA, large for gestational age), which is often the cause of perinatal injuries [5]. Depending on the type of diabetes this complication concerns $15 \%$ to even $50 \%$ of pregnant women with type 1 diabetes [6].

Fetuses of mothers with pregestational diabetes mellitus are particularly vulnerable to complications during pregnancy and the percentage of pregnancies complicated by excessive growth continues to increase despite improved diabetes management [1]. 
Fetal overgrowth is challenging to predict and capture early during pregnancy. It affects mainly children exposed in utero to maternal hyperglycemia, followed with fetal hyperinsulinemia. Moreover, studies are suggesting the involvement of other factors including maternal lipids, adipokines and excessive weight gain [7]. These newborns are also at risk of obesity, diabetes and cardiovascular disease later in life $[8,9]$. Most of the fetal complications are irreversible, so capturing the earliest moment when growth disorders occur to prevent this process is essential.

Due to the still inaccurate ultrasound diagnostics and severe consequences for the neonatal health that result from undetected fetal overgrowth, special attention should be placed on the best obstetric and diagnostics procedures for diabetic pregnant women to prevent them.

Our work aimed to assess the incidence of disturbances in the fetal growth in the studied group of patients with pre-existing type 1 diabetes and to analyze the ultrasound growth charts of fetuses from the examined group of pregnant women to indicate the period of pregnancy in which they begin. The next goal is to identify factors that significantly affect fetal growth in pregnant women with diabetes.

Spotting of this moment and identifying factors influencing the fetal growth disturbances would possibly allow identifying a group of women particularly predisposed to these complications.

\section{MATERIAL AND METHODS}

In line with the Polish standards of medical care each woman with pregestational diabetes was admitted to our department the tertiary reference centre for pregnant diabetic women from central-west Poland, as soon as her primary care gynaecologist confirmed the pregnancy.

We collected data from 200 pregnancies with pregestational type 1 diabetes mellitus (PGDM1) that we admitted in the first trimester to our department in 2015-2018. Patients were regularly admitted in the clinic in every trimester to assess health status, metabolic control, and to supervise the fetal development. During every visit, each patient underwent an ultrasound examination by an experienced sonographer according to a standard protocol. In the study, based on biparietal diameter (BPD), head circumference $(\mathrm{HC})$, abdominal circumference $(\mathrm{AC})$ and femur length (FL) measurements, fetal weight was estimated according to the Hadlock 3 formula, which is considered to be the most accurate estimation of fetal weight in diabetic pregnancies [2].

We analyzed data of diabetic, singleton pregnancies we retrospectively retrieved from our department's database. All women conceived naturally. Analyzed patients were admitted to our department at least once before the $12^{\text {th }}$ week of pregnancy, according to the last menstrual period (LMP). Gestational age was confirmed or corrected in the first trimester of pregnancy with the measurement with transvaginal ultrasound. Each pregnant woman with type 1 diabetes admitted to the department we thoroughly interviewed during the first antenatal visit. Only patients admitted in the first trimester were taken into the study.

Patients were all Caucasian and received standard pregnancy care for patients with diabetes, as recommended by the Polish Diabetes Association and Polish Gynaecological Society. As target we took a fasting glucose level of 3.9-5 mmol/L (70-90 mg/dL), 1-hour postprandial glucose below $7.8 \mathrm{mmol} / \mathrm{L}$ (140 $\mathrm{mg} / \mathrm{dL})$, and glycated haemoglobin ( $\mathrm{HbA} 1 \mathrm{c})$ below $6.5 \%$ (48 mmol/L) in the first trimester of pregnancy and next trimesters below $6.0 \%$ (42 mmol/L) [4].

At the first antenatal visit we recorded the following maternal parameters: maternal age, duration and class of diabetes according to White, pre-pregnancy body mass index (BMI), the concentration of haemoglobin A1c (HbA1c), presence of vascular complications (hypertension, proteinuria, and retinopathy) and pregnancy planning.

All women were on intensive insulin therapy using either multiple daily injections (MDI) or continuous subcutaneous insulin infusion (CSII).

Exclusion criteria from this paper were multiple pregnancies, miscarriage, preeclampsia and delivery before completed the $34^{\text {th }}$ week of gestation or delivery in another hospital.

We collected anthropometric, clinical and laboratory data during three planned hospital admissions, according to our protocol: in the first trimester $\left(<12^{\text {th }}\right.$ week of gestation), in mid-pregnancy $\left(20^{\text {th }}-24^{\text {th }}\right.$ weeks of pregnancy) and before delivery $\left(34^{\text {th }}-39^{\text {th }}\right.$ weeks of gestation).

The term large-for-gestational-age we used for fetuses or newborns with estimated weight above the $90^{\text {th }}$ percentile or more than two standard deviations from the mean for the gestational age. Small for gestational age refers to fetuses or neonates that were born with weight below the $10^{\text {th }}$ centile or more than two standard deviations from the mean for the gestational age adjusted to World Health Organization definitions [5].

Blood samples were taken for analysis after overnight, in the fasting state and immediately transported to the central laboratory of the Gynaecologic Obstetrical University Hospital in Poznan. HbA1c level in the whole blood was determined using the turbid metric inhibition immunoassay, Tina-quant Haemoglobin A1c II test in a Cobas c311 analyzer (Roche Diagnostics, Basel, Switzerland). The normal range for this test is $4.8-6.0 \%(29-42 \mathrm{mmol} / \mathrm{mol})$ for a non-pregnant population. The total serum cholesterol, high density lipoprotein (HDL)-cholesterol and triglyceride (TG) levels were determined with Roche Diagnostics reagents (Cholesterol CHODPAP, HDL-C plus and Triglycerides GPO-PAP, respectively) on a Cobas c501 analyzer. 


\begin{tabular}{|c|c|c|c|c|}
\hline & $\begin{array}{c}\text { AGA } \\
n=131\end{array}$ & $\begin{array}{c}\text { LGA } \\
n=60\end{array}$ & $\begin{array}{l}\text { SGA } \\
n=9\end{array}$ & $\mathbf{p}^{*}$ \\
\hline Diabetes duration [years] (SD) & $10.8(7.7)$ & $11.2(7.5)$ & $9.2(6.1)$ & 1 \\
\hline Mean age of DM diagnosis [years] (SD) & $19.3(9.1)$ & $17.2(8.5)$ & $18.5(8.7)$ & 0.9 \\
\hline Body weight before pregnancy [kg] (SD) & $73.1(18.2)$ & $70(17.4)$ & $90.2(14.4)$ & 0.09 \\
\hline Weight gain during pregnancy [kg] (SD) & $11.9(5.7)^{1}$ & $14.7(4.7)^{1}$ & $11.7(4.9)$ & 0.5 \\
\hline Weight gain in $1^{\text {st }}$ trimester [kg] (SD) & $1.1(2.2)$ & $0.8(3.1)$ & $1.4(1.8)$ & 0.4 \\
\hline Weight gain in $2^{\text {nd }}$ trimester [kg] (SD) & $4.7(3.7)$ & $5.7(3.8)$ & $3.4(3.1)$ & 0.7 \\
\hline Weight gain in $3^{\text {rd }}$ trimester [kg] (SD) & $5.5(4.3)$ & $7.0(5.1)$ & $5.3(2.6)$ & 0.7 \\
\hline
\end{tabular}

*Kruskal-Walis and ANOVA tests comparing three study groups; ${ }^{1}$ comparing two groups AGA vs LGA with Mann-Whitney test ( $\left.p<0.05\right)$; SD — standard deviation; SGA - small for gestational age; AGA - appropiate for gestational age; GA- gestational age

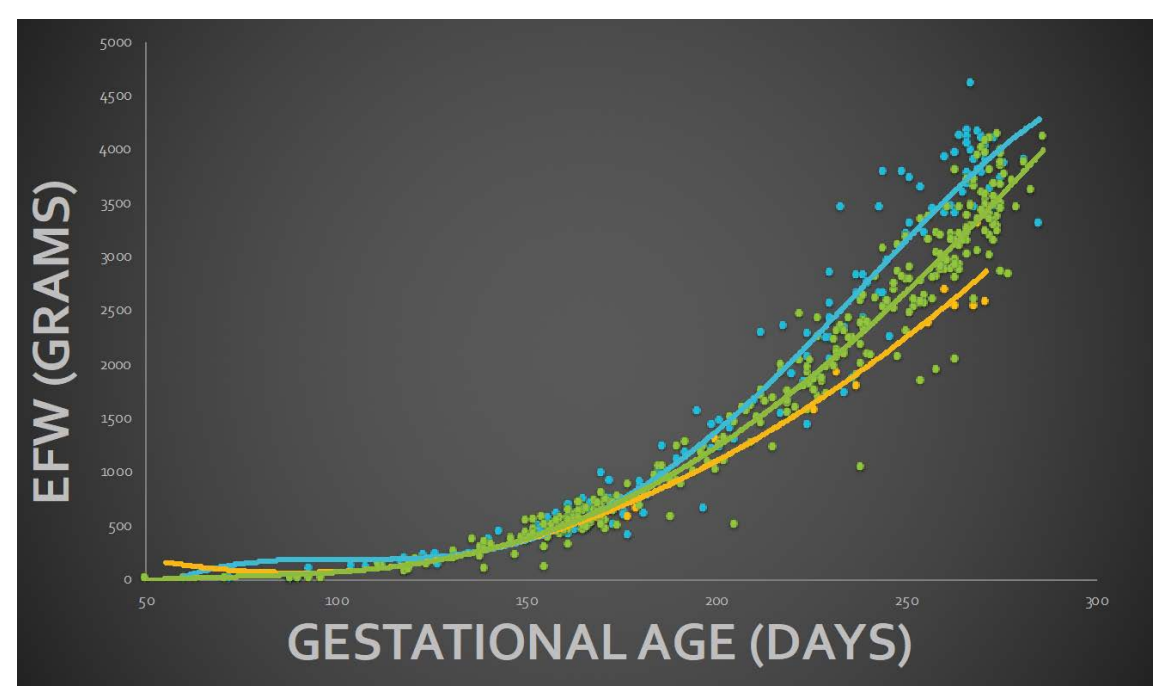

Figure 1. Estimated fetal weight in ultrasound according to the final weight of the newborns - three groups: SGA (yellow), AGA (green), LGA (blue) SGA — small for gestational age; AGA — appropiate for gestational age; LGA — large for gestational age

The following formula we used to calculate the level of low-density lipoprotein (LDL) [LDL-cholesterol = total cholesterol-HDL-cholesterol-(TG/5)]. Total cholesterol (CHOL), LDL-cholesterol (LDL-CH), HDL-cholesterol (HDL-CH), and triglycerides (TG) levels were measured with the appropriate Roche Diagnostics reagents (cholesterol CHOD-PAP, HDL-C plus and triglycerides GPO-PAPrespectively) on Hitachi 912 analyzer. Reference values for $\mathrm{CHOLis} 3.9-7.2 \mathrm{mmol} / \mathrm{L}$, TG is $0.46-1.71 \mathrm{mmol} / \mathrm{L}$, for LDL-CH is $4.1 \mathrm{mmol} / \mathrm{L}$ and for $\mathrm{HDL}-\mathrm{CH}<1.29 \mathrm{mmol} / \mathrm{L}$ for women.

We performed statistical analysis using PQstat program. The Shapiro-Wilik test we used for testing the normality of data distribution. We used the ANOVA test to check the significance of the difference between three groups, if data fitted normal distribution. Results are as the mean \pm standard deviation (SD). Comparisons of non-normally distributed data are using the Kruskal-Walis test with conclusions expressed as the median and interquartile range (IQR). Parameters that shown significant differences in ANOVA and
Kruskal-Walis tests were further analyzed with post-hoc analysis with approppriately Fisher LSD and Dunn Bonferroni tests to find the differences between the subgroups.

\section{RESULTS}

In our 200 of PGDM1 group, sixty (30\%) patients gave birth to children meeting LGA criteria, and nine (4.5\%) newborns were small for gestational age. The birth weight of the remaining newborns $(n=131,65.5 \%)$ was appropriate for the gestational age (AGA). Maternal parameters related to the bodyweight of the newborn divided into three groups (LGA, AGA, and SGA), are presented in the Table 1.

The Figure 1 shows fetal growth charts in these three subgroups of patients (AGA, SGA, LGA). We present subsequent ultrasound examinations on a scatter plot with a superimposed trend line, which maps the trajectory of fetal growth in selected groups.

Data has shown that both estimated fetal weight and abdominal circumference start to differ between our three 


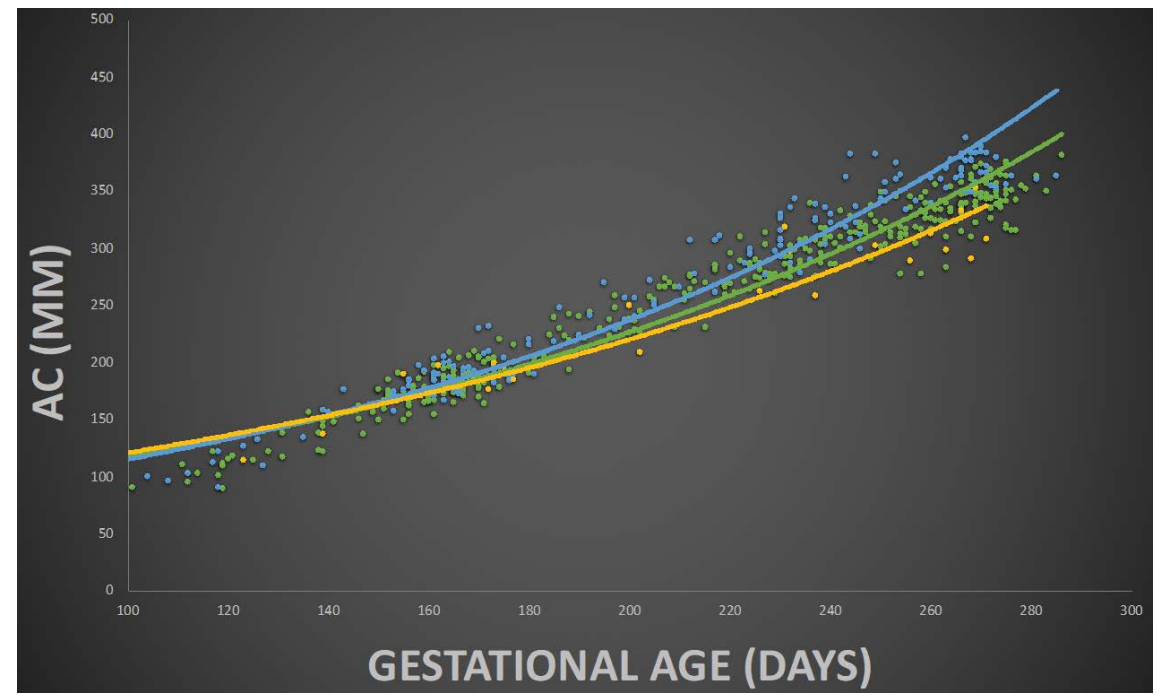

Figure 2. Abdominal circumference (AC) in ultrasound according to the final weight of the newborns - three groups: SGA (yellow), AGA (green), LGA (blue) SGA — small for gestational age; AGA — appropiate for gestational age; LGA — large for gestational age

groups around the $23^{\text {rd }}$ week (161 days). Similar results were presented by other authors [10]. Abdominal circumference is a measurement that varies the most the fetuses with growth disorders, as well as with excessive as with too small fetal growth. In our diabetic group we observed differences in abdominal circumference, especially in the LGA fetuses in the third trimester (Fig. 2).

In the next part of our study we analyzed the metabolic parameters of the patients to show which of them had a significant impact on the development of excessive fetal growth. Our results have shown, that there was no difference in any of the studied parameters between patients who delivered LGA and AGA newborns and all of them met the target criteria of glycemic control. The percentage of $\mathrm{HbA} 1 \mathrm{c}$, which reflects the mean glycemia was not significantly different between the LGA and AGA group. SGA patients had higher $\mathrm{HbA} 1 \mathrm{c}$ levels in the first trimester, but this difference was not significant (Tab. 2). In none of the trimesters $\mathrm{HbA} 1 \mathrm{c}$ concentrations were statistically different between LGA, SGA, and AGA patients.

Also the concentration of the maternal lipids concentrations (HD-L, LDL-, total cholesterol and triglycerides) analyzed in each of the three trimesters did not differ significantly between diabetic patients with excessive and with proper fetal growth. We found somewhat surprising differences between the SGA and the AGA groups. SGA patients had a substantially higher level of the total cholesterol, LDL-cholesterol and triglycerides in the first trimester $(p<0.05)$. They also needed more insulin in the first and second trimester to maintain euglycemia $(p=0.05)$. Interesting differences we found in weight gain during pregnancy $(p<0.05)$ Women who delivered LGA neonates presented higher weight gain during pregnancy than AGA group.
For the analysis of the effectiveness of fetal weight prediction before delivery we qualified 87 patients whose ultrasound was performed up to seven days before childbirth. We predicted in 69 (79\%) a weight as being appropriate for gestational age. After birth this diagnosis was confirmed in $59(68 \%)$ newborns. Eight newborns (9\%) turned out to be LGA, while two belonged to the SGA group. Predicting LGA based on EFW was less sensitive than based on abdominal circumference. Our results revealed that $\mathrm{AC}$ measurements had higher accuracy in LGA detecting and lower negative predictive value, which is crucial to avoid perinatal injuries in diabetic pregnancies (Tab. 4, 5)

\section{DISCUSSION}

Pregestational diabetes may lead to many maternal complications during pregnancy but especially coincides with the development of adverse neonatal outcomes. One of the most common complications is excessive fetal growth, which can be associated with fetal fetopathy including metabolic disturbances and the risk of shoulder dystocia [4].

There is no one specific formula for fetal weight estimation in small for gestational age fetuses as well as in big babies. Coombs et al. [11] compared 31 formulas in their study and did not show significant superiority of individual methods. Many authors have proved that Hadlock's formula is the best for estimating the weight of hypotrophic fetuses, as well as the macrosomic babies. Therefore we decided to use it for our measurements [12].

Excessive fetal growth increases the risk of cesarean section and traumatic delivery [5]. To predict and avoid these complications precise estimating of fetal weight in ultrasound is necessary. Also very important is to find the moment when fetal overgrowth is starting, because then 


\begin{tabular}{|c|c|c|c|c|}
\hline & $\begin{array}{c}\text { AGA } \\
n=131\end{array}$ & $\begin{array}{c}\text { LGA } \\
n=60\end{array}$ & $\begin{array}{l}\text { SGA } \\
n=9\end{array}$ & $\mathbf{p}^{*}$ \\
\hline $\mathrm{HbA} 1 \mathrm{c} 1^{\text {st }}$ trimester [\%] (SD) & $6.9(1.6)$ & $6.5(1.1)$ & $7.1(1.8)$ & 0.2 \\
\hline $\mathrm{HbA} 1 \mathrm{c} 2^{\text {nd }}$ trimester [\%] (SD) & $5.7(0.7)$ & $6.8(8.1)$ & $5.3(0.8)$ & 0.5 \\
\hline $\mathrm{HbA1c} 3^{\text {rd }}$ trimester [\%] (SD) & $6.0(0.7)$ & $5.9(0.8)$ & $6.1(1.4)$ & 0.9 \\
\hline $\mathrm{HDL} 1^{\text {st }}$ trimester $[\mathrm{mg} / \mathrm{dL}](\mathrm{SD})$ & $68.6(17.1)$ & $70.3(17.5)$ & $60.7(22.3)$ & 0.5 \\
\hline $\mathrm{HDL} 2^{\text {nd }}$ trimester $[\mathrm{mg} / \mathrm{dL}](\mathrm{SD})$ & $85.1(19.0)$ & 89.0 (19.6) & $73.9(9.6)$ & 0.2 \\
\hline $\mathrm{HDL} 3^{\text {rd }}$ trimester [mg/dL] (SD) & $76.8(19.2)$ & $79.4(20.3)$ & $71.4(22.1)$ & 0.7 \\
\hline LDL $1^{\text {st }}$ trimester $[\mathrm{mg} / \mathrm{dL}](\mathrm{SD})$ & $85.3(24.1)^{1}$ & $79.1(20.6)^{6}$ & $100.5(35.4)^{1.6}$ & 0.01 \\
\hline LDL $2^{\text {nd }}$ trimester $[\mathrm{mg} / \mathrm{dL}](\mathrm{SD})$ & $127.4(96.8)$ & $120.1(35.1)$ & $124.4(34.6)$ & 0.9 \\
\hline LDL $3^{\text {rd }}$ trimester $[\mathrm{mg} / \mathrm{dL}](\mathrm{SD})$ & $146.5(54.0)$ & $140.7(42.3)$ & $148.6(29.6)$ & 0.8 \\
\hline Total cholesterol $1^{\text {st }}$ trimester [mg/dL] (SD) & $170.1(27.4)^{2}$ & $163.3(25.1)^{7}$ & $186.7(39.8)^{2.7}$ & 0.01 \\
\hline Total cholesterol $2^{\text {nd }}$ trimester [mg/dL] (SD) & $230.1(41.2)$ & $233.7(51.2)$ & $248.4(39.4)$ & 0.7 \\
\hline Total cholesterol $3^{\text {rd }}$ trimester [mg/dL] (SD) & $269.3(67.5)$ & $296.0(231.1)$ & $260.5(46.6)$ & 0.9 \\
\hline $\mathrm{TG} 1^{\text {st }}$ trimester $[\mathrm{mg} / \mathrm{dL}](\mathrm{SD})$ & $83.1(44.4)$ & $69.8(29.5)^{5}$ & $127.6(68.0)^{5}$ & 0.01 \\
\hline $\mathrm{TG} 2^{\text {nd }}$ trimester [mg/dL] (SD) & $148.5(70.0)$ & $142.3(52.3)$ & $194.1(56.8)$ & 0.3 \\
\hline $\mathrm{TG} 3^{\text {rd }}$ trimester [mg/dL] (SD) & $248.8(89.6)$ & $219.4(93.2)$ & $258.9(86.9)$ & 0.2 \\
\hline Hypertensionn (\%) & $25(19.2)$ & $10(16.7)$ & $2(22.2)$ & 0.7 \\
\hline Proteinuria $1^{\text {st }}$ trimester $\mathrm{n}(\%)$ & $13(12.3)$ & $8(17.4)$ & 0 & 0.4 \\
\hline Proteinuria $2^{\text {nd }}$ trimester $\mathrm{n}(\%)$ & $12(18.1)$ & $3(10.7)$ & 0 & 0.4 \\
\hline Presence of proteinuria $3^{\text {rd }}$ trimester $n(\%)$ & $15(6.6)$ & $8(38.1)$ & $1(20)$ & 0.9 \\
\hline Insulin intake $1^{\text {st }}$ trimester [IU] (SD) & $38.0(17.4)^{3}$ & $30.0(17.4)^{3.8}$ & $48.6(25.7)^{8}$ & 0.01 \\
\hline Insulin intake $2^{\text {nd }}$ trimester [IU] (SD) & $42.0(18.5)^{4.9}$ & 37.1 (19.5) & $68.0(33.7)^{4.9}$ & 0.01 \\
\hline Insulin intake $3^{\text {rd }}$ trimester [IU] (SD) & $34.3(18.1)$ & $30.4(15.1)$ & $38.8(23.5)$ & 0.4 \\
\hline $\begin{array}{l}\text { Insulin intake per kg } \\
\text { I trimester [IU/kg] (SD) }\end{array}$ & $0.49(0.28)$ & $0.53(0.22)$ & $0.51(0.35)$ & 0.5 \\
\hline
\end{tabular}

*Kruskal-Walis and ANOVA tests comparing three study groups; ${ }^{1,2,3,4,6,7,8,9}$ POST-HOC statistically significant difference $\mathrm{p}<0.05$. Fisher LSD test; ${ }^{5} \mathrm{POST}-\mathrm{HOC}$ statistically significant difference $\mathrm{p}<0.05$. Dunn Bonferroni test; SD — standard deviation; SGA — small for gestational age; AGA — appropiate for gestational age; GA — gestational age; $\mathrm{HDL}$ - high density lipoprotein; $\mathrm{LDL}$ - low-density lipoprotein; TG — triglyceride

we have the possible chance to intensify the metabolic and maternal weight gain control.

In our study we tried to determine the week of pregnancy when excessive fetal weight gain begins. In daily clinical practise we observe that children who are macrosomic after delivery, start their excessive weight gain around the $26^{\text {th }}-28^{\text {th }}$ weeks of pregnancy. Determining the growth trajectories for fetuses with AGA, LGA and SGA in our studied groups we noticed the tendency to diverge curves distinctly earlier, i.e. from 24-25 weeks of pregnancy. In our study, we estimated the moment when fetal growth impairment begins among patients with pregestational diabetes. However statistical analyzes have not confirmed this. They have shown that based on the available data we are not able to determine the exact moment when the excessive growth of the fetus or its restriction begins. We speculate that our studied group, however well treated with proper metabolic control is quite heterogeneous and it might impair the precision of this finding. Patients differ in duration of the disease, weight gain during pregnancy, total insulin doses, presence of vascular and renal complications and this complexity of the diabetic disease potentially causes a different moment of the beginning of fetal growth disturbances.

The proper on time detection of this process might give in impulse for better glycemic and weight gain control, which in some studies confirmed the suppressing of fetal overgrowth [13]. Our observations might constitute a motivation for searching for better indicators of growth impairment in the early stages of pregnancy. From all measured metabolic parameters in our studied group only maternal weight gain during pregnancy differed significantly the groups with LGA and AGA newborns. It shows that higher energy intake determines not only maternal weight gain but also affects fetal weight. In the SGA group patients were heavier at the beginning of pregnancy, had significantly higher triglycerides in I and II trimester and insulin intake during the second trimester. Surprisingly only two patients from that group presented hypertension during pregnancy. 


\begin{tabular}{|c|c|c|c|c|}
\hline & $\begin{array}{c}\text { AGA } \\
\text { Mean (SD) } \\
n=131\end{array}$ & $\begin{array}{c}\text { LGA } \\
\text { Mean (SD) } \\
n=60\end{array}$ & $\begin{array}{c}\text { SGA } \\
\text { Mean (SD) } \\
n=9\end{array}$ & $\mathbf{p}^{*}$ \\
\hline $\begin{array}{l}\text { BPD } \\
\text { (22-24 week) } \\
\text { mm (SD) }\end{array}$ & $55.1(3.4)$ & $55.8(3.3)$ & $53.2(5.7)$ & 0.8 \\
\hline $\begin{array}{l}\mathrm{HC} \\
\text { (22-24 week) } \\
\mathrm{mm} \text { (SD) }\end{array}$ & $203.4(11.3)$ & $208.3(11.2)$ & $190.3(23.4)$ & 0.3 \\
\hline $\begin{array}{l}\text { AC } \\
\text { (22-24 week) } \\
\mathrm{mm} \text { (SD) }\end{array}$ & $178.1(12.9)$ & $184.2(11.9)^{1}$ & $165.5(20.6) 1$ & 0.03 \\
\hline $\begin{array}{l}\mathrm{FL} \\
\text { (22-24 week) } \\
\mathrm{mm} \text { (SD) }\end{array}$ & $39.1(2.9)$ & $40.2(3.2)$ & $34.5(8.3)$ & 0.6 \\
\hline $\begin{array}{l}\text { BPD } \\
\text { (29-31 week) } \\
\text { mm (SD) }\end{array}$ & $78.2(6.2)$ & $79.4(8.5)$ & 0 & 0.7 \\
\hline $\begin{array}{l}\mathrm{HC} \\
\text { (29-31 week) } \\
\mathrm{mm} \text { (SD) }\end{array}$ & $273.1(41)$ & $261.1(58.8)$ & 0 & 0.5 \\
\hline $\begin{array}{l}\text { AC } \\
\text { (29-31 week) } \\
\text { mm (SD) }\end{array}$ & $256.8(39.2)$ & $256.2(58)$ & 0 & 1 \\
\hline $\begin{array}{l}\mathrm{FL} \\
\text { (29-31 week) } \\
\mathrm{mm} \text { (SD) }\end{array}$ & $60.7(10)$ & $62(14.5)$ & 0 & 0.8 \\
\hline $\begin{array}{l}\text { BPD } \\
\text { (36-38 week) } \\
\mathrm{mm} \text { (SD) }\end{array}$ & $85.6(2.9)^{2}$ & $92.7(4.1)^{2.5}$ & $87.5(0.2)^{5}$ & 0.0004 \\
\hline $\begin{array}{l}\mathrm{HC} \\
(36-38 \text { week) } \\
\mathrm{mm}(\mathrm{SD})\end{array}$ & $323.1(8.8)^{3}$ & $335.6(12.7)^{3.6}$ & $320.5(8.4)^{6}$ & 0.00001 \\
\hline $\begin{array}{l}\text { AC } \\
\text { (36-38 week) } \\
\mathrm{mm} \text { (SD) }\end{array}$ & $325.7(20)^{4.8}$ & $350.7(16.5)^{4.7}$ & $302.7(11)^{7.8}$ & $<0.000001$ \\
\hline $\begin{array}{l}\text { FL } \\
\text { (36-38 week) } \\
\text { mm (SD) }\end{array}$ & $70(3.5)$ & $72.2(2.7)$ & $53.5(35.7)$ & 0.1 \\
\hline \multicolumn{5}{|c|}{$\begin{array}{l}\text { *- Kruskal-Walis and ANOVA tests comparing three study groups } \\
\text { 1,3,4,6,7, } \text { POST- HOC statistically significant difference } p<0,05 \text {. Fisher } L S D \text { test. } \\
\text { 2,5.-POST- HOC statistically significant difference } p<0,05 \text {. Dunn Bonferroni test. } \\
\text { SD - standard deviation; SGA - small for gestational age; AGA - appropiate for gestational age; GA - gestational age; BPD - biparietal diameter; HC - gead } \\
\text { circumference; AC - abdominal circumference; FL - femur lenght }\end{array}$} \\
\hline
\end{tabular}

\begin{tabular}{|c|c|c|c|}
\hline & born LGA & born AGA & sum \\
\hline predicted LGA & 12 & 1 & 13 \\
\hline predicted AGA & 8 & 59 & 67 \\
\hline sum & 20 & 60 & 80 \\
\hline \multicolumn{2}{|c|}{ sensitivity } & \multicolumn{2}{|c|}{$60.00 \%$} \\
\hline \multicolumn{2}{|c|}{ specifity } & \multicolumn{2}{|c|}{$98.33 \%$} \\
\hline \multicolumn{2}{|c|}{ Positive predictive value } & \multicolumn{2}{|c|}{$92.31 \%$} \\
\hline \multicolumn{2}{|c|}{ Negative predictive value } & \multicolumn{2}{|c|}{$88 \%$} \\
\hline
\end{tabular}

LGA — large for gestational age; AGA — appropiate for gestational age 
Table 5. Prediction of the occurrence of large for gestational age based on AC

\begin{tabular}{|c|c|c|c|}
\hline & born LGA & born AGA & sum \\
\hline predicted $A C \geq 90$ percentile & 16 & 3 & 19 \\
\hline predicted $\mathrm{AC}<90$ percentile & 4 & 57 & 61 \\
\hline sum & 20 & 60 & 80 \\
\hline \multicolumn{2}{|c|}{ sensitivity } & \multicolumn{2}{|c|}{$80.00 \%$} \\
\hline \multicolumn{2}{|c|}{ specifity } & \multicolumn{2}{|c|}{$95.00 \%$} \\
\hline \multicolumn{2}{|c|}{ Positive predictive value } & \multicolumn{2}{|c|}{$84.00 \%$} \\
\hline \multicolumn{2}{|c|}{ Negative predictive value } & \multicolumn{2}{|c|}{$93.00 \%$} \\
\hline
\end{tabular}

LGA — large for gestational age; AGA — appropiate for gestational age; AC — abdominal circumference

Still, as we know parameters mentioned above belong to the group of metabolites that predispose to changes in placental vessels and could cause insufficient placental circulation resulting in growth restriction.

Our results confirmed that in women with suspicion of LGA we may estimate it more precisely, using abdominal circumference calculation, than using the estimated fetal weight. Abdominal circumference above the $90^{\text {th }}$-centile has higher negative predictive value in detecting LGA than the estimation of fetal weight, which allows the obstetrician to plan the route of delivery with higher accuracy. Our results are not in concordance with Blue, who observed that after the $24^{\text {th }}$ week of gestation AC measurement has no advantage in the LGA risk assessment in fetuses with estimated overgrowth [14]. Caradeux has not confirmed the effectiveness of longitudinal growth assessment as a better method to detect excessive growth compared to standard biometric measurements [15]. In our opinion although fetal weight estimation is still imperfect there is no better way, as longitudinal observations to diagnose hypertrophy among fetuses. It is in line with Ben-Haroush [16].

Accurate estimation of fetal weight is fundamental both to predict the occurrence of complications and also involves the choice of delivery method. In the study of Phillips et al. the effectiveness of ultrasound performed two weeks before delivery in predicting macrosomia was only 33\% [17]. Their study shows how unpredictable can be the weight of the fetus and how dynamically it changes, especially in the last weeks of pregnancy, in which we decide about the method of delivery. Ben-Haroush in his review concludes that based on the available methods we are not able to determine the birth weight of fetuses accurately in women with diabetes [16]. His observation makes the choice of delivery more challenging and therefore increasing the risk of shoulder dystocia. In our study we estimated the risk of macrosomia using known risk factors. We took into consideration prior history of macrosomia, maternal pre-pregnancy weight, weight gain during pregnancy, multiparity, fetal sex (male), gestational age (40 weeks), maternal birth weight, and ma- ternal height and obesity [17]. We confirmed that the total maternal weight gain in a relatively well-controlled diabetic group predisposes significantly to fetal overgrowth.

The main goal of our study was to indicate the moment when growth disorders begin to develop. Schaefer-Graf et al. revealed that the following factors were leading in the late second and early third trimesters, genetic and patient's history turned out to be the most significant factors influencing fetal growth. In the late third-trimester maternal hyperglycemia has the most significant influence on the macrosomia [18]. In our group of patients the maternal weight gain throughout pregnancy was the only statistically significant factor that correlated with macrosomia.

We assume that it is the matter of vital importance not to stop looking for new parameters to diagnose both excessive growth and growth restriction as soon as possible during pregnancy. Especially in the group of patients with the disease predisposing to these complications like with pregestational diabetes. Our results confirmed that in patients with an efficient metabolic control the next steps should be done. Strict weight gain control during pregnancy, planning the pregnancy by reducing the body weight and treatment of hypertriglyceridemia before pregnancy might let to avoid impaired fetal growth. Maybe further studies will find some vascular complications in early pregnancy, putting such patients to a group of the high risk of developing growth restriction.

\section{REFERENCES}

1. López-Tinoco C, Jiménez-Blázquez JL, Larrán-Escandón L, et al. Effect of Different Insulin Therapies on Obstetric-Fetal Outcomes. Sci Rep. 2019; 9(1): 17650, doi: 10.1038/s41598-019-54164-4, indexed in Pubmed: 31776421.

2. Aviram A, Yogev Y, Ashwal E, et al. Prediction of large for gestational age by various sonographic fetal weight estimation formulas-which should we use? J Perinatol. 2017; 37(5): 513-517, doi: 10.1038/.jp.2017.5, indexed in Pubmed: 28151496.

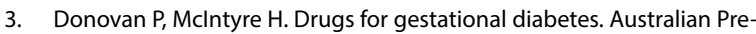
scriber. 2010; 33(5): 141-144, doi: 10.18773/austprescr.2010.066.

4. Wender-Ożegowska E, Bomba-Opoń D, Brazert J, et al. Standards of Polish Society of Gynecologists and Obstetricians in management of women with diabetes. Ginekol Pol. 2018; 89(6): 341-350, doi: 10.5603/GP.a2018.0059, indexed in Pubmed: 30010185. 
5. Kiserud T, Piaggio G, Carroli G, et al. The World Health Organization Fetal Growth Charts: A Multinational Longitudinal Study of Ultrasound Biometric Measurements and Estimated Fetal Weight. PLoS Med. 2017; 14(1): e1002220, doi: 10.1371/journal.pmed.1002220, indexed in Pubmed: 28118360.

6. Ladfors L, Shaat N, Wiberg N, et al. Fetal overgrowth in women with type 1 and type 2 diabetes mellitus. PLoS One. 2017; 12(11): e0187917, doi: 10.1371/journal.pone.0187917, indexed in Pubmed: 29121112.

7. Gutaj P,Wender-Ożegowska E, Brązert J. Maternal lipids associated with large-for-gestational-age birth weight in women with type 1 diabetes: results from a prospective single-center study. Arch Med Sci. 2017; 13(4): 753-759, doi: 10.5114/aoms.2016.58619, indexed in Pubmed: 28721142.

8. Mourad M, Friedman AM, Ajemian B, et al. Fetal growth velocity in diabetics and the risk for shoulder dystocia: a case-control study. J Matern Fetal Neonatal Med. 2019 [Epub ahead of print]: 1-5, doi: 10.1080/14767058.2019.1651838, indexed in Pubmed: 31370705.

9. Turkmen S, Johansson S, Dahmoun M. Foetal Macrosomia and Foetal-Maternal Outcomes at Birth. J Pregnancy. 2018; 2018: 4790136, doi: 10.1155/2018/4790136, indexed in Pubmed: 30174954.

10. Júnior EA, Peixoto A, Zamarian A, et al. Macrosomia. Best Practice \& Research Clinical Obstetrics \& Gynaecology. 2017;38:83-96, doi: 10.1016/j. bpobgyn.2016.08.003.

11. Combs CA, Rosenn B, Miodovnik M, et al. Sonographic EFW and macrosomia: is there an optimum formula to predict diabetic fetal macrosomia? J Matern Fetal Med. 2000; 9(1): 55-61, doi: 10.1002/(SICI)1520-6661(200 001/02)9:1<55::AID-MFM12>3.0.CO;2-9, indexed in Pubmed: 10757437.

12. Aviram A, Yogev Y, Ashwal E, et al. Different formulas, different thresholds and different performance-the prediction of macrosomia by ultrasound.
J Perinatol. 2017; 37(12): 1285-1291, doi: 10.1038/jp.2017.134, indexed in Pubmed: 28906497.

13. Schaefer-Graf UM, Kjos SL, Kilavuz O, et al. Determinants of fetal growth at different periods of pregnancies complicated by gestational diabetes mellitus or impaired glucose tolerance. Diabetes Care. 2003; 26(1): 193-198, doi: 10.2337/diacare.26.1.193, indexed in Pubmed: 12502680.

14. Blue NR, Yordan JM, Holbrook BD, et al. Abdominal Circumference Alone versus Estimated Fetal Weight after 24 Weeks to Predict Small or Large for Gestational Age at Birth: A Meta-Analysis. Am J Perinatol. 2017; 34(11): 1115-1124, doi: 10.1055/s-0037-1604059, indexed in Pubmed: 28672412

15. Caradeux J, Eixarch E, Mazarico E, et al. Second- to Third-Trimester Longitudinal Growth Assessment for the Prediction of Largeness for Gestational Age and Macrosomia in an Unselected Population. Fetal Diagn Ther. 2018; 43(4): 284-290, doi: 10.1159/000477460, indexed in Pubmed: 28719900.

16. Ben-Haroush $A$, Yogev $Y$, Hod M. Fetal weight estimation in diabetic pregnancies and suspected fetal macrosomia. J Perinat Med. 2004; 32(2): 113-121.

17. Phillips AM, Galdamez AB, Ounpraseuth $\mathrm{ST}$, et al. Estimate of fetal weight by ultrasound within two weeks of delivery in the detection of fetal macrosomia. Aust N Z J Obstet Gynaecol. 2014; 54(5): 441-444, doi: 10.1111/ajo.12214, indexed in Pubmed: 24773582.

18. Perlow JH, Morgan MA, Montgomery $\mathrm{D}$, et al. Perinatal outcome in pregnancy complicated by massive obesity. Am J Obstet Gynecol. 1992; 167(4 Pt 1): 958-962, doi: 10.1016/s0002-9378(12)80019-6, indexed in Pubmed: 1415432. 